Rev. salud pública. 14 sup (2): 69-85, 2012

\title{
Efectos de la entrevista motivacional sobre la motivación autónoma en jóvenes consumidores de alcohol
}

\section{The effect of motivational interviews on young drinkers' autonomous motivation}

\author{
Luis Flórez-Alarcón ${ }^{1}$ y Carol A. Castellanos-Morales²
}

1 Facultad de Ciencias Humanas, Universidad Nacional de Colombia. Bogotá, Colombia. luis@ florez. info, cacastellanosm@unal.edu.co

Recibido 25 Mayo 2011/Enviado para Modificación 8 Agosto 2011/Aceptado 3 Noviembre 2011

\section{RESUMEN}

Objetivo Evaluar los cambios en la motivación autónoma y controlada en un grupo de adolescentes consumidores en riesgo y consumidores excesivos de alcohol, que recibió una intervención basada en la Entrevista Motivacional (EM).

Métodos Se implementó una intervención grupal basada en la EM para observar sus efectos sobre los tipos de motivación. Se conformaron dos grupos, un grupo experimental, y un grupo control en un diseño pretest-postest. Participaron en total 63 adolescentes, 31 conformaron el grupo experimental, ( $80 \%$ hombres) y 32 el grupo control (68 \% hombres). La motivación autónoma y la motivación controlada se evaluaron mediante el TSRQ.

Resultado El análisis de varianza muestra que los puntajes de la motivación autónoma no presentaron cambios estadísticamente significativos después de la intervención, mientras que la motivación controlada sí los presentó $(p<.05)$, sugiriendo que la intervención promovió este tipo de regulación hacia el consumo moderado, expresada en términos de controles externos, por ejemplo, la aprobación social.

Conclusión Estos resultados sugieren que la EM es una técnica promisoria para la intervención con adolescentes colombianos ya que tiene efectos positivos cuantificables sobre los dos tipos de motivación y probablemente la orientación motivacional hacia el control medió los resultados. Es importante considerar en el futuro la orientación motivacional individual y el género.

Palabras Clave: Consumo de bebidas alcohólicas, motivación, adolescencia, autonomía personal (fuente: DeCS, BIREME). 


\section{ABSTRACT}

Objective Assessing changes in autonomous and controlled motivation in a group of young at risk consumers and excessive alcohol consumers receiving motivational interviewing-based (MI) Intervention.

Methods Ml-based group intervention was put into practice to observe its effects on types of motivation. Two groups were formed (an experimental and a control group) using pretest-posttest design. 63 adolescents participated: 31 formed the experimental group ( $80 \%$ male) and 32 the control group (68\% male). The treatment self-regulation questionnaire (TSRQ) was used to evaluate autonomous and controlled motivation.

Results Analysis of variance revealed that autonomous motivation scores had no statistically significant change after the brief intervention, whereas this was so with controlled motivation $(p<.05)$, suggesting that brief intervention had promoted this type of regulation towards moderate consumption expressed in terms of an external control, such as gaining social approval.

Conclusion The above results suggested that $\mathrm{MI}$ is a promising technique for intervention with Colombian adolescents as it had measurable positive effects on both types of motivation and motivational orientation toward control probably mediated the results. Individual motivational orientation and gender should be considered in the future.

Key Words: Motivational interviewing, autonomous motivation, controlled motivation, adolescent drinking, brief interventions (source: $M e S H, N L M$ ).

$\mathrm{E}$ consumo de alcohol en Colombia es un fenómeno de interés a nivel institucional gubernamental, a nivel investigativo y a nivel de la sociedad en general debido a los hallazgos al caracterizarlo en la población joven.

El estudio de Pérez y Scoppetta (1) muestra que en Colombia el inicio del consumo se encuentra alrededor de los 11 años, siendo en los varones mas temprano y de mayor intensidad, el $86,7 \%$, de los encuestados en las capitales declaró haber consumido bebidas alcohólicas alguna vez en su vida, el $67 \%$, haberlo hecho en el último año, el 27, $9 \%$, en el último mes y el $13 \%$, en la última semana. A nivel general, el 34,3\% declara no tomar, el 42,8 \% consume menos de seis veces al año, el 14,1 consume todos o casi todos los meses, el 3,7 \%, consume varias veces a la semana y el 0,4 $\%$, toma todos o casi todos los días; Este mismo trabajo evidencio como situaciones problemáticas: haberse emborrachado (30,9\%), vómito $(23,7$ $\%)$, peleas en casa por beber $(18,8 \%)$, pelear con la pareja $(16,3 \%)$, siendo estos hechos más comunes para los hombres que para las mujeres. 
El Estudio Nacional de Consumo de Sustancias Psicoactivas en Colombia 2008 encontró que en el grupo de 12 a 17 años el $20 \%$ de los encuestados reconoce consumo en el último mes, llegando al $46 \%$ en el grupo de 18 a 24 años, mientras que el porcentaje de jóvenes entre 12 y 17 años que reporta consumo de riesgo o perjudicial es de un $6,1 \%$.

Como respuesta a estos indicadores, los modelos motivacionales de explicación y de intervención han tomado fuerza, entre ellos se encuentra la teoría de la autodeterminación (TAD), (2-4) que propone que todas las conductas yacen alrededor de un continuo que va desde la heteronomía o regulación externa, a la autonomía o verdadera autorregulación, este continuo resulta de los diferentes tipos de fuerzas que pueden movilizar a las personas hacia la acción, generandose motivación extrínseca por fuerzas tales como recibir una recompensa o evitar el castigo por el comportamiento y motivación intrínseca cuando se promueve desde el interior e incluye entre otros el interés personal (Cuadro 1). Adicionalmente la TAD plantea que la salud psicológica requiere la satisfacción de tres necesidades: competencia, relación y autonomía (3-5), esta última se refiere al beneficio de experimentar un sentido de elección y autoría con respecto a la conducta y se relaciona con el deseo de las personas de autoorganizar y autogobernar su experiencia y comportamiento, tomar decisiones informadas basadas en una conciencia de las propias necesidades y valores (6).

Cuadro 1. Continúo de Autodeterminación

\begin{tabular}{|c|c|c|c|c|c|}
\hline Conducta & \multicolumn{2}{|c|}{ No auto determinada } & & \multicolumn{2}{|c|}{ Autodeterminada } \\
\hline Motivación & Amotivación & & Extrínseca & & Intrínseca \\
\hline $\begin{array}{l}\text { Estilos de } \\
\text { regulación }\end{array}$ & Sin regulación & Externa & Introyectada Identificada & Integrada & Intrínseca \\
\hline $\begin{array}{l}\text { Locus de } \\
\text { causalidad }\end{array}$ & Impersonal & Externo & Algo externo Algo interno & Interno & Interno \\
\hline $\begin{array}{l}\text { Procesos } \\
\text { reguladores } \\
\text { relevantes }\end{array}$ & $\begin{array}{l}\text { No intencional } \\
\text { no valorado } \\
\text { incompetencia } \\
\text { falta de control }\end{array}$ & $\begin{array}{c}\text { Obediencia } \\
\text { recompensas } \\
\text { externas } \\
\text { castigos }\end{array}$ & $\begin{array}{cc}\text { Autocontrol } & \\
\text { implicación } & \text { Importancia } \\
\text { ego } & \text { persona } \\
\text { recompensas } & \text { valoración } \\
\text { internas } & \text { consciente } \\
\text { castigos } & \\
\end{array}$ & $\begin{array}{c}\text { Congruencia } \\
\text { conciencia } \\
\text { síntesis con } \\
\text { uno mismo }\end{array}$ & $\begin{array}{l}\text { Interés disfrute } \\
\text { Satisfacción } \\
\text { inherente }\end{array}$ \\
\hline
\end{tabular}

Fuente: Adaptada de Ryan y Deci (7)

La TAD utiliza el término internalización para describir el grado en el cual las conductas que no se disfrutan totalmente se convierten en autónomas por medio de su incorporación en las estructuras personales como una expresión de sus valores y corresponden a aquellas conductas con 
las que el individuo se compromete con voluntad y autonomía mediante un proceso de cambio activo que le permite crecer y ser saludable (8).

Actualmente, hay una distinción entre la motivación autónoma y la motivación controlada, la primera es entendida como combinación de regulación identificada y regulación intrínseca, que puede estar basada en identificaciones bien internalizadas, incorporación de las conductas a los valores personales con un sentido de volición, de libertad y elección, la vivencia de un sentido de auto aprobación y autopromoción (9). por ejemplo, un consumidor de sustancias que decide entrar al tratamiento porque estima que buscar ayuda es congruente con el valor personal de tratar todo lo posible para vivir un estilo de vida libre de drogas (6). Por su parte, la motivación controlada, carece de integración y el individuo es presionado para actuar, sea por imposición externa (complacer el entorno) o por fuerzas internas (ansiedad o culpa), este es el caso de un consumidor que entra al tratamiento porque se adhiere al control de sus padres. Por supuesto que la regulación autónoma resulta más estable y duradera y por ende tiene mayores efectos positivos en el bienestar de las personas (7), mostrando mejores resultados en el uso terapeutico en eventos como el alcohol, la pérdida de peso, el ejercicio, la diabetes, el tabaco, la adherencia persistente a la medicación, el ajuste a VIH y el autocuidado (10). En poblaciones clínicas se ha encontrado que la motivación interna se asocia positivamente con mejores resultados y las personas que presentaron motivación interna y externa tuvieron más probabilidad de continuar su tratamiento (5). Además los hallazgos han sugerido que los terapeutas que emplean estrategias más autónomas influyen directamente en la motivación al tratamiento (6).

Con respecto al consumo de alcohol entre adolescentes el apoyo a la autonomía se ha asociado con tener valores intrínsecos más fuertes, lo que los protege del consumo (11), mientras que en estudiantes universitarios se encuentra que la baja autonomía y ser susceptible al control se relaciona con las expectativas positivas hacia el alcohol y su consumo problemático (12, 13). Además la orientación al control promueve la motivación extrínseca para reducir indicadores visibles de preocupación por el consumo (14).

En este contexto la entrevista motivacional (EM) es una estrategia de intervención psicológica centrada en la persona, diseñada para resolver la ambivalencia e incrementar la estimulación al cambio, a partir de conceder un lugar privilegiado a la relación interpersonal (15). Las estrategias de la 
EM se adhieren a un enfoque centrado en la automotivación y el apoyo, planteando cinco principios:

1. Expresar empatía: Mostrar una actitud de comprensión y de aceptación utilizando la escucha reflexiva;

2. Crear una discrepancia: entre la conducta actual y unos objetivos vitales más amplios (la salud, el éxito profesional, la estabilidad familiar y de pareja, la carrera, el deporte o, el estatus social);

3. Evitar la discusión: ya que puede llevar a una lucha por aceptar quién tiene la razón y no aumenta la motivación hacia un cambio positivo;

4. Darle un giro a la resistencia: cambiar las percepciones de la persona con respecto a las razones para no cambiar, examinando nuevas perspectivas y nueva información;

5. Fomentar la autoeficacia: aumentar las percepciones de las personas sobre su capacidad para hacer frente a los obstáculos y tener éxito en el cambio (15).

Las intervenciones que se utilizan en la EM han evidenciado impacto en el funcionamiento social $\mathrm{y}$, consecuencias positivas para un rango amplio de situaciones más allá de los problemas específicos del comportamiento (16). Es un método breve y efectivo para ser aplicado a cambios en el abuso de sustancias, particularmente para la población adolescente dada su sensibilidad y resistencia a los intentos adultos de controlar o dirigir su comportamiento (17) igualmente se ha señalado que los participantes evaluan positivamente una intervención individual basada en la EM (18, 19), siempre y cuando se identifiquen las motivaciones subyacentes para que las intervenciones sean exitosas (20), por lo que se ha sugerido la posible complementariedad de la TAD y la EM aplicadas para el cambio de comportamientos relacionados con la salud $(14,21,22)$ que consiste en el fomento del cambio al promover la integración de la regulación de una nueva conducta (moderación en el consumo de alcohol), de tal manera que la persona este comprometida por voluntad propia y de acuerdo con sus metas y valores (21).

El papel más importante de la EM en su relación con la TAD se refiere al establecimiento de un contexto terapéutico que apoya la necesidad de autonomía por medio de sus principios y estilo. Así el factor social central que influencia la motivación (23) se encuentra abordado por la EM para contribuir a la motivación autónoma. 
Por lo tanto, este trabajo busca establecer cuáles son los cambios que se presentan en un grupo de adolescentes consumidores en riesgo y consumidores excesivos de alcohol, en cuanto a la motivación autónoma al cambio (representada en razones autónomas) luego de recibir una intervención basada en la EM.

\section{METODOLOGIA}

Diseño

El programa se estructuró para ser aplicado dentro de un diseño cuasiexperimental de grupo control pretest-postest con lista de espera, el cual comprende un grupo experimental y otro de control con dos aplicaciones de la intervención para completar la muestra, donde ambos recibieron una medición pre-intervención y una post-intervención.

\section{Participantes}

La muestra se compuso de estudiantes de secundaria de dos colegios distritales de Bogotá de dos localidades, de grados $9^{\circ}, 10^{\circ}$ y $11^{\circ}$ tanto mujeres como hombres interesados en participar en charlas acerca del consumo del alcohol y que puntuaran 2 o 3 en la prueba CAGE. Se tomaron estos cursos porque son aquellos en los que se manifiesta con más claridad el inicio del proceso de abuso de alcohol (24). Se excluyeron de la muestra jóvenes con problemas recurrentes de consumo de alcohol o posible abuso según lo reportaban las orientadoras. Se asumió un muestreo al azar dentro de las jornadas académicas (mañana y tarde) pues estas representan unidades enteras asignadas a los grupos experimental y control respectivamente (28). A los participantes y a sus padres se les entregó un formato de consentimiento informado de su asistencia al programa que fue debidamente diligenciado.

\section{Instrumentos}

Cuestionario de línea de base. Incluyó datos demográficos: edad, género, nivel educativo y el CAGE (29), utilizado para detectar abuso de alcohol y dependencia, que está compuesto por cuatro preguntas de respuesta dicotómica sí o no. Las preguntas se centran en el acrónimo CAGE y cada una corresponde a una letra: "C" 'Cutting down' (Reflexiones personales sobre la necesidad de disminuir su consumo), "A" "Annoyance by criticism' (críticas de terceros con relación a su hábito de consumo), "G" 'Guilty feeling' (presencia de sentimientos de culpabilidad por los daños 
provocados por el consumo), y "E" 'Eye-openers' (necesidad de consumo matutino). El cuestionario proporciona una puntuación total que se obtiene sumando las puntuaciones en los 4 ítems. En cada ítem, la respuesta afirmativa vale un punto y la negativa cero.

Se utilizó la clasificación de Oswald (30): 0-1 consumo no perjudicial, 2 consumo de riesgo, 3 consumo excesivo y 4 dependencia alcohólica, según el cual un puntaje de 2 o más en esta prueba establece el límite que divide el consumo sin riesgo del consumo de riesgo. El puntaje 3 se consideraría predictor de un consumo excesivo, y el puntaje 4 predictor de un consumo dependiente (31).

A nivel internacional el CAGE ha probado ser un instrumento válido para el tamizaje de problemas de alcohol en poblaciones clínicas. En el estudio de Flórez-Alarcón (32), con la población bogotana se encontró una buena confiabilidad del CAGE (0.7) y un buen resultado de validez concurrente con los puntajes del CID (Cuestionario de Indicadores Diagnósticos) (0.60), Es un instrumento poco intimidante, corto y puede ser administrado en menos de 60 segundos.

TSRQ-alcohol. Cuestionario de autorregulación para el tratamiento (Treatment Self- Regulation Questionnaire (TSRQ)(9, 33)para la medición de la motivación autónoma. Este instrumento se relaciona con las razones por las cuales las personas desempeñan o desempeñarían algún comportamiento saludable, en este caso consumir alcohol moderadamente. Este instrumento tiene el propósito de medir el grado en el cual la motivación de una persona hacia un comportamiento particular es relativamente autónoma. El instrumento tiene tres subescalas que pueden usarse independientemente: el estilo de regulación autónoma, el estilo de regulación controlada y la amotivación (que se refiere a estar poco motivado). El TSRQ ha sido descrito y validado teóricamente por medio de análisis de invarianza, análisis factoriales exploratorios y confirmatorios; y cuenta con una consistencia interna aceptable $\alpha>0,73(9)$. El instrumento sigue una modalidad tipo likert con 7 puntos que va desde 'no es cierto en lo absoluto' a 'muy cierto', algunos ítems pueden verse en la Tabla 4.

Generalmente, las respuestas a los ítems relacionados con la autonomía se promedian para formar el reflejo de la motivación autónoma hacia el comportamiento que es materia de estudio. En resumen, el instrumento evalúa 
las razones autónomas para el cambio que darían cuenta de la progresión de la persona a lo largo del continuo hacia la integración de la conducta. La escala tiene 15 ítems: 6 evalúan la motivación autónoma, 6 evalúan la motivación controlada, y 3 evalúan amotivación.

El uso de este instrumento en el presente estudio requirió su adaptación al español y el análisis de su consistencia y de su validez para la población que hizo parte de la intervención, esta puede ser consultada en Castellanos (25). Sin embargo como resultado de la adaptación, se conservaron algunos ítems para el análisis de resultados (ver Tabla 1).

Procedimiento

La intervención fue aplicada por dos psicólogos, con experiencia previa de mínimo 12 horas de entrenamiento en las técnicas de la EM de acuerdo con los estándares en esta materia (34). Además se sostuvieron cuatro reuniones previas a la aplicación de la intervención para conocer a fondo su estructura basados en la descripción detallada del programa. Estas reuniones incluyeron la retroalimentación mutua, la colaboración de un experto guía, el reforzamiento positivo de las respuestas consistentes con la EM, resolución de problemas y dificultades y la práctica de habilidades de EM en juegos de roles.

Cuadro 2 . Ítems seleccionados para el análisis con los grupos control y experimental

\begin{tabular}{l} 
Subescala \\
\hline Porque siento que deseo tomar responsabilidad de mi propia salud \\
Porque personalmente creo que es lo mejor para mi salud \\
Motivaciónporque lo he pensado cuidadosamente y creo que es muy importante para \\
Autónoma muchos aspectos de mi vida \\
Porque es una decisión importante que en verdad deseo tomar \\
Porque es consistente con las metas que tengo en mi vida \\
Porque es muy importante para estar tan saludable como sea posible \\
Porque los demás se molestarían si no lo hiciera \\
Motivación \\
Controlada Porque siento presión de los demás para hacerlo \\
Porque quiero que los demás vean que si puedo hacerlo
\end{tabular}

Posteriormente se pasóa la fase de selección de los participantes que consistió en contactar a la sección de orientación de dos colegios distritales de Bogotá por medio de una reunión de presentación de la investigación, con el fin de obtener el espacio de invitar a los participantes. Luego de concertar un espacio 
dentro de la jornada académica, se convocó en las aulas a los participantes de grados $9^{\circ}, 10^{\circ}$ y $11^{\circ}$ de secundaria a diligenciar el cuestionario de línea de base luego de informarles que hacía parte de un proyecto de investigación acerca del consumo de alcohol. Luego los jóvenes fueron invitados a participar en el programa, se enfatizó que su participación era voluntaria y que sus resultados serían confidenciales. La siguiente semana se seleccionaron los adolescentes que, según la prueba CAGE, fueran consumidores en riesgo y consumidores excesivos de alcohol; a los estudiantes seleccionados se les entregó un formato de consentimiento informado para su asistencia al programa para que lo diligenciaran y uno para sus padres que fue devuelto en el transcurso de la semana.

Los grupos control y experimental se conformaron según la propuesta de asignar unidades enteras (28), la conformación se hizo mediante la selección al azar de 60 personas para cada grupo de una muestra por cada jornada académica de aquellos que tuvieron puntuaciones mayores o iguales a 2 en el cuestionario CAGE.

El grupo que recibió la intervención, se citó tres semanas después de la primera reunión para comenzar el programa que constó de 12 horas divididas en sesiones semanales de 2 horas. Se hicieron dos aplicaciones de la intervención para completar la muestra, no fueron simultáneas por asuntos logísticos en los colegios, pero sí tuvieron la misma duración y el procedimiento en ambos colegios fue igual. Los temas a abordar en el programa incluyeron: aplicación del TSRQ, la información y la exploración de los efectos del alcohol, retroalimentación sobre el consumo personal, identificación de situaciones de riesgo, análisis de riesgos y consecuencias del consumo, balance decisional, solución de problemas y planificación del autocontrol. Durante toda la intervención se utilizó el estilo de la EM en sus cinco principios al explorar percepciones, valores, y metas de los estudiantes para activar la motivación al cambio. El grupo control no recibió intervención alguna durante las semanas de duración de la intervención y permaneció en lista de espera mientras el grupo experimental sí la recibió; luego de este período de tiempo, en un segundo momento cuatro semanas después, los dos grupos recibieron la prueba post intervención. Por razones éticas, luego de terminar el estudio se aplicó la intervención al grupo control.

En el análisis de los resultados se tuvieron en cuenta, dentro del grupo experimental, los datos de aquellos jóvenes que participaron durante mínimo 4 
sesiones. Por ello la muestra final fue de 66 jóvenes, 35 en el grupo control que fueron seleccionados al azar de los 60 iniciales y 31 en el grupo experimental, este grupo presentó menos participantes ya que el contacto con los estudiantes de último grado (15 personas) no se puedo realizar y algunos participantes de otros grados decidieron no asistir al taller por temor a ser etiquetados como 'alcohólicos' ya que fue difícil controlar las actitudes de los docentes al ser informados del taller y porque los docentes no daban permiso para salir al taller.

\section{RESULTADOS}

Los grupos control y experimental en la medición pre intervención mostraron ser homogéneos tanto a nivel de características sociodemográficas tales como el género $X^{2}(1, \mathrm{~N}=66)=1,25, p>0,05$ y la edad $X^{2}(5, \mathrm{~N}=66)=0,83, p>0,05$; así como en la distribución según el grado de noveno a once $X^{2}(2, \mathrm{~N}=66)=2,58$, $p>0,05$. Igualmente fueron muestras similares en cuanto a las mediciones de motivación autónoma y controlada, $t(64)=-0,62, p>.05$ y $t(64)=-0,57 ; p>0,05$. Las medias de las mediciones preintervención de motivación en ambos grupos aparecen en la Tabla 1.

Tabla 1. Medias y desviación típica en la medición pre de la motivación

\begin{tabular}{lcccc}
\hline & \multicolumn{3}{c}{ Motivación autónoma } & Motivación controlada \\
& M & DT & M & DT \\
\hline \multicolumn{1}{c}{ Grupo control } & & & & \\
Total & 3,46 & 1,64 & 2.37 & 1.37 \\
Mujeres & 3,40 & 1,87 & 2.13 & 1.62 \\
Hombres & 3,49 & 1,57 & 2.47 & 1.27 \\
$\quad$ Grupo Experimental & & & & \\
Total & 3,71 & 1,56 & 2.58 & 1.57 \\
Mujeres & 2,94 & 1,17 & 1.33 & 0.43 \\
Hombres & 3,90 & 1,60 & 2.88 & 1.60 \\
\hline
\end{tabular}

Nota. $\mathrm{N}=66$

Es importante anotar que para los análisis siguientes el grupo control estuvo compuesto por 32 participantes ya que no se pudo contactar a tres estudiantes para la prueba post. Los datos sociodemográficos de los participantes en el estudio aparecen en la Tabla 2.

Motivación Autónoma y Controlada

Se encontró que hubo diferencias significativas en las medias de los puntajes de motivación autónoma $\mathrm{t}(30)=-3,48, \mathrm{p}=0,00$ y de motivación controlada $\mathrm{t}$ 
$(30)=-2,65, p=0,13$ en el grupo experimental luego de recibir la intervención. Por su parte el grupo control no mostró diferencias significativas en los puntajes de la motivación autónoma t $(31)=-1,07, \mathrm{p}=0,29$ y la motivación controlada t $(31)=0,43, p=0,63$.

Tabla 2. Datos sociodemográficos de los participantes

\begin{tabular}{lcc}
\hline & Grupo Control & Grupo Experimental \\
\hline Género & & \\
Hombres & 22 & 25 \\
Mujeres & 10 & 6 \\
Nivel educativo & & \\
Noveno & 14 & 15 \\
Décimo & 11 & 14 \\
Once & 7 & 2 \\
Edad (media) & 15.41 & 15.48 \\
Edad (rango) & $13-18$ & $13-18$ \\
CAGE & & \\
2 & 19 & 27 \\
3 & 13 & 4 \\
\hline
\end{tabular}

Se realizó un ANOVA de medidas repetidas para explorar si la intervención tuvo un efecto significativo tanto en la motivación autónoma como en la motivación controlada en el grupo experimental.

Figura 1. Perfil para la Motivación Autónoma

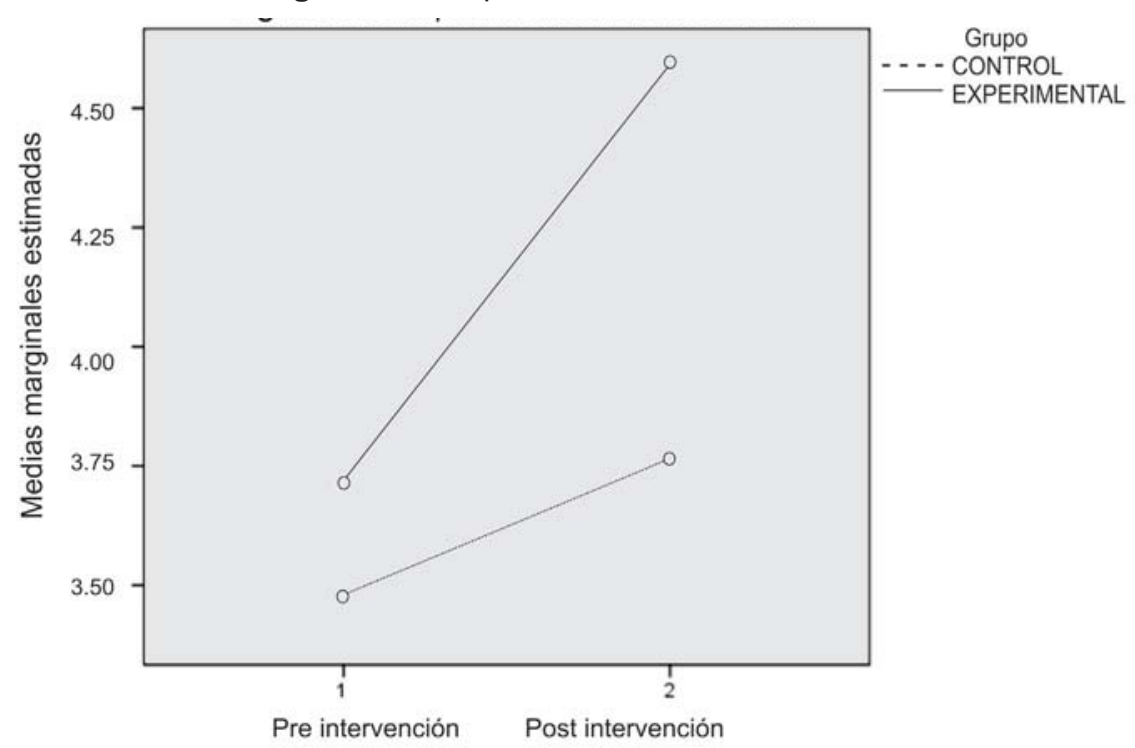


Utilizando los estadísticos univariados, a nivel intrasujetos se encontraron diferencias significativas en las medias de las puntuaciones de los participantes al comparar las mediciones pre intervención y post intervención $\mathrm{F}(1,61)=9,13$, $p=0,00, \eta^{2}=0,13$, es decir se evidenció un efecto del tiempo y de la medición sobre la motivación autónoma. Sin embargo no existió un efecto significativo de la interacción entre los grupos control y experimental y las puntuaciones en la motivación autónoma $\mathrm{F}(1,61)=2,35, p=0,13, \eta^{2}=0,03$. Además al observar las pruebas intersujetos, el efecto del factor grupo no fue significativo $\mathrm{F}$ $(1,61)=2,20, p=0,14, \eta^{2}=0,03$, por tanto no se puede afirmar que haya diferencias entre los grupos con respecto a los puntajes de motivación autónoma pre y post intervención.

La figura 1 muestra el gráfico de perfil para las mediciones de motivación autónoma. En el caso de la motivación controlada, igualmente con la aproximación univariada, no se encontraron diferencias significativas en las medias de las puntuaciones entre las mediciones pre y post intervención $\mathrm{F}(1,61)=3,09, p=0,08$, $\eta^{2}=0,04$. No obstante hubo un efecto significativo de la interacción entre el factor grupo (control vs experimental) sobre la motivación controlada $F(1,61)=5,36$, $p=0,02, \eta^{2}=0,08$. Igualmente se observaron las pruebas intersujetos donde el efecto del factor grupo fue significativo $\mathrm{F}(1,61)=4,67, p=0,03, \eta^{2}=0,07$, por tanto, se puede afirmar que hay diferencias entre los grupos con respecto a los puntajes de motivación controlada. La figura 2 muestra el gráfico de perfil para las mediciones de motivación controlada donde es más claro que la motivación controlada mostró un aumento en el grupo experimental y una interacción con el factor grupo.

Figura 2. Perfil para la Motivación Controlada

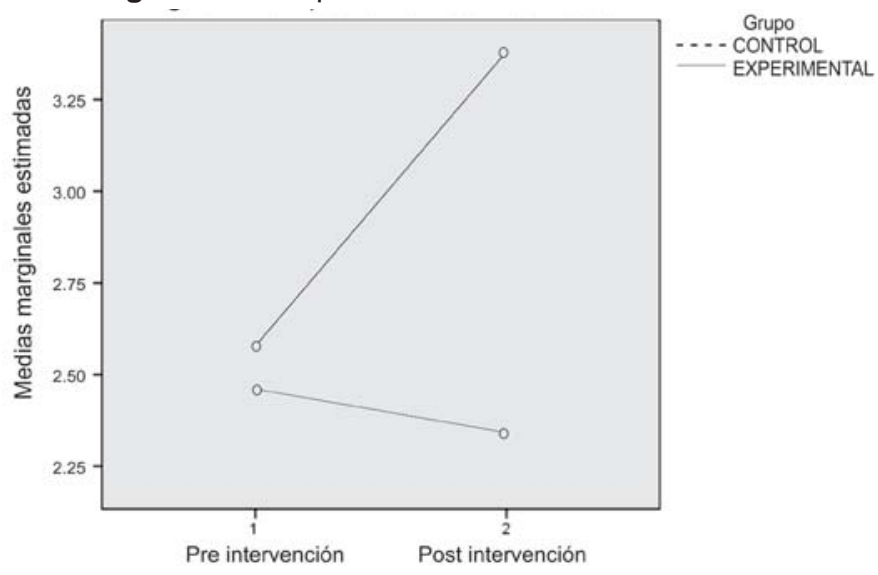




\section{DISCUSIÓN}

La motivación autónoma aumentó de manera significativa en el grupo experimental cuando se compararon los resultados de manera intragrupal pero no alcanzo niveles de significancia estadística, lo cual quizá puede ser explicado por una sensibilización pretest (28) para el grupo control pues en este grupo la motivación autónoma presentó un perfil de aumento. Sin embargo los resultados con respecto a la motivación controlada despiertan un mayor interés ya que se evidencio un incremento significativo. Las hipótesis de trabajo recogidas, hacían preveer un efecto de la intervención sobre la motivación autónoma, es decir, sobre las razones de tipo interno, basadas en valores y decisiones personales para consumir moderadamente, sin embargo, el cambio observado luego de la intervención en la motivación controlada sugiere, en primer lugar, una posible influencia de las diferencias individuales sobre las razones para consumir 'responsablemente', es decir que la intervención probablemente promovería en algunos participantes razones de tipo más externo para cambiar el patrón de consumo dependiendo de su manera de motivarse (por medio del control vs. autonomía), así que vale la pena explorar este tipo de orientaciones en los adolescentes teniendo en cuenta que en este estudio la motivación controlada muestra influencia en las razones para no beber, además porque en poblaciones universitarias se ha encontrado la influencia de esta orientación sobre la motivación al consumo (12).

Igualmente, el cambio presentado en la motivación controlada se puede relacionar con los efectos que tiene informar a los participantes acerca de las consecuencias negativas asociadas al consumo de alcohol y brindar retroalimentación normativa, pues tal información tiene mayor influencia hacia la reducción del consumo de alcohol en aquellas personas con orientación controlada (14). Específicamente, algunos temas de la intervención realizada en este trabajo: los efectos del alcohol sobre los adolescentes y la exposición de las cifras de consumo, promoverían razones extrínsecas para reducir indicadores que preocupan como es 'hacer el ridículo', involucrarse en relaciones afectivas no deseadas, enfermarse al otro día de haber bebido y 'no acordarse de la noche anterior'; aspectos que los participantes expresaron como una inquietud generalizada. Es así como queda abierta la cuestión referente a la influencia de las diferencias de la orientación motivacional sobre la reducción de conductas de riesgo. 
No es del todo extraño el efecto de la sobre la motivación controlada, ya que los adolescentes dan alto grado de importancia a la imagen, el dinero o la fama como aspiraciones extrínsecas para justificar el consumo de alcohol (11). Por ello es importante recordar uno de los ítems que evaluaron la motivación controlada, "consumiría 'responsablemente' porque deseo la aprobación de los demás", ya que en la respuesta a estos ítems se ven reflejadas tales aspiraciones lo que indica que estas juegan un papel importante en las decisiones de los jóvenes sobre sus conductas de riesgo tanto para comprometerse en ellas como para disminuirlas. Además, al remitirse a las actividades del taller, se encuentra que la referencia a sus grupos sociales (familia y amigos) y a los valores de amor y amistad fue una constante y se relaciona directamente con los resultados en la motivación controlada.

Por otro lado, el incremento de las razones extrínsecas para moderar el consumo evidencia los beneficios del fomento de la motivación controlada. Los análisis sugieren que ambos tipos de motivación aumentan: la controlada y la autónoma, esto representa un mayor beneficio para los participantes, evidente en la conciencia sobre los riesgos de continuar un patrón de consumo excesivo y en el compromiso para mantener el cambio, manifestado en la última sesión. Concretamente, el beneficio de la presencia de ambos tipos de motivación ha sido planteado en estudios referentes al tratamiento e intervención en el área de consumo de alcohol y otras drogas (35); pues existe mayor probabilidad de continuar el tratamiento cuando la motivación de tipo externo está acompañada de motivación interna. En este sentido, los resultados de este trabajo son alentadores en términos de promover una adopción de razones para moderar el consumo de alcohol que son tanto externas como internas; por supuesto, es necesario utilizar métodos para confirmar que ambos tipos de motivación influyen en el compromiso al cambio y el mantenimiento del mismo.

Es necesario anotar que en el tiempo diseñado para la intervención no se logró fortalecer significativamente la motivación autónoma cuando se compararon los dos grupos. En consecuencia se sugiere que los próximos trabajos que se hagan en este tema, incluyan evaluaciones de seguimiento que den cuenta de la calidad de la motivación fomentada en la intervención.

La implicación principal del presente estudio es que se encuentra evidencia preliminar para vincular la TAD con la EM, pues ambos tipos 
de motivación son procesos susceptibles de modificación mediante la EM en este grupo de adolescentes. Por tanto este trabajo contribuye a abrir la investigación de las intervenciones en la población adolescente que como afirman Grenard et al. (2006) es un campo nuevo de investigación.

Además los resultados de este trabajo indican que la calidad de los dos tipos de motivación, según lo plantea la TAD, es fomentada por la EM. Así, se muestra que debe hacerse una diferenciación de los resultados que se obtengan al realizar intervenciones basadas en la EM, pues la calidad de la motivación que podría dar cuenta del cambio y del mantenimiento del mismo puede ser de diferente tipo en la población adolescente, como sucedió en este trabajo donde los dos tipos de calidad de la motivación cambian como resultado de la intervención. Así, los resultados de este trabajo pueden empezar a aclarar qué pasa con la motivación hacia la moderación en el consumo con los adolescentes. En consecuencia, posteriores trabajos podrían evaluar las orientaciones individuales y el impacto de cada componente de la EM por separado para observar sus efectos en personas con diferentes orientaciones.

Una de las limitaciones de este estudio fue el número de participantes que no permite hacer una generalización importante empero, sí sugiere nuevos temas de investigación, por ejemplo el examen de las diferencias en cuanto a los resultados de estar motivado por control y estar motivado por la autonomía. Además la presencia reducida de mujeres en la muestra, implica que el análisis de los resultados en las mujeres es poco representativo, por lo tanto, se sugiere estudiar las intervenciones basadas en la EM con este grupo ya que los patrones de consumo riesgoso y excesivo en Colombia incluyen cada vez más a las mujeres y es importante aclarar cuáles son los tipos de motivación para reducir el consumo en esta población.

De otro lado, el medio escolar presenta varias dificultades para la aplicación de intervenciones de este tipo como es la poca información a los docentes quienes no sabían que los estudiantes estaban participando en la investigación y retrasaban o no permitían la salida del aula. Igualmente existe la dificultad del manejo de las etiquetas con los participantes a pesar de advertir a la institución de esta barrera. Con respecto a la medición de las variables, es claro que este trabajo evaluó principalmente las razones para consumir alcohol moderadamente para apreciar los tipos de motivación autónoma y controlada. Sin embargo, no se hizo un evaluación del 
comportamiento de consumo que diera cuenta de la frecuencia e intensidad de consumo antes y después de la intervención, aunque esto haría más complejo el diseño, sería de gran valor incluir esta evaluación en próximos trabajos

\section{REFERENCIAS}

1. Pérez A, Scopetta O. Consumo de alcohol en menores de 18 años en Colombia: 2008 un estudio con jóvenes escolarizados de 12 a 17 años en 7 capitales de departamento y dos municipios pequeños. Bogotá: Corporación Nuevos Rumbos; 2008.

2. Deci EL, Ryan RM. Self-determination theory: A macrotheory of human motivation, development, and health. Canadian Psychology 2008; 49(3):182-5.

3. Ryan RM, Deci EL. Intrinsic and Extrinsic Motivations: Classic Definitions and New Directions. Contemporary educational psychology. 2000; 25(1):54-67.

4. Deci EL, Ryan RM. The "what" and "why" of goal pursuits: Human needs and the selfdetermination perspective. Psychological Inquiry 2000; 11:227-68.

5. Ryan RM. Psychological needs and the facilitation of integrative processes. Journal of Personality.1995; 63(3):397-427.

6. Klag S. Self Determination Theory and the Theory of Planned Behaviour Applied to Substance Abuse Treatment in a Therapeutic Community Setting [Thesis Doctoral]. Brisbane, Australia: Universidad de Griffith; 2006.

7. Ryan RM, Deci EL. Self-determination theory and the facilitation of intrinsic motivation, social development, and well-being. American psychologist. 2000; 55(1):68-78.

8. Williams GC, McGregor HA, Sharp D, Levesque C, Kouides RW, Ryan RM, et al. testing a self-determination theory intervention for motivating tobacco cessation: Supporting autonomy and competence in a clinical trial. Health Psychology. 2006; 25(1):91-101.

9. Williams GG, Gagné M, Ryan RM, Deci EL. Facilitating autonomous motivation for smoking cessation. Health Psychology. 2002; 21(1):40.

10. Ryan RM, Patrick H, Deci EL, Williams GC. Facilitating health behaviour change and its maintenance: Interventions based on Self-Determination Theory. The European Health Psychologist. 2008; 10:2-5.

11. Williams GC, Hedberg VA, Cox EM, Deci EL. Extrinsic Life Goals and Health-Risk Behaviors in Adolescents1. Journal of Applied Social Psychology. 2000; 30(8):1756-71.

12. Neighbors C, Lewis MA, Fossos N, Grossbard JR. Motivation and risk behaviors: A selfdetermination perspective. In: Brown L, editor. Psychology of Motivation. New York: Nova Science Publishers; 2007.p. 99.

13. Neighbors C, Walker DD, Larimer ME. Expectancies and Evaluations of Alcohol Effects among College Students: Self-Determination as a Moderator*. Journal of studies on alcohol. 2003; 64(2):292-301.

14. Neighbors $C$, Lewis MA, Bergstrom RL, Larimer ME. Being controlled by normative influences: Self-determination as a moderator of a normative feedback alcohol intervention. Health psychology: official journal of the Division of Health Psychology, American Psychological Association. 2006; 25(5):571.

15. Miller W, Rollnick S. La Entrevista Motivacional. Barcelona: The Guilford Press; 1999.

16. Burke BL, Arkowitz H, Menchola M.The efficacy of motivational interviewing: a meta-analysis of controlled clinical trials. Journal of Consulting and Clinical Psychology.2003; 71(5):843-60.

17. Masterman PW, Kelly AB. Reaching adolescents who drink harmfully: Fitting intervention to developmental reality. Journal of Substance Abuse Treatment. 2003; 24(4):347-55.

18. Grenard J, Ames S, Wiers R, Thush C, Stacy A, Sussman S. Adolescent Health Brief Intervention for Substance Use among At-Risk Adolescents: A Pilot Study. Journal of Adolescent Health. 2007; 40:188-91. 
19. Marlatt GA, Witkiewitz K. Harm reduction approaches to alcohol use:: Health promotion, prevention, and treatment. Addictive behaviors.2002; 27(6):867-86.

20. Grenard JL, Ames SL, Pentz MA, Sussman S. Motivational interviewing with adolescents and young adults for drug-related problems. Int J Adolesc Med Health. 2006; 18(1):53-67.

21. Markland D, Ryan RM, Tobin VJ, Rollnick S. Motivational interviewing and selfdetermination theory. Journal of Social and Clinical Psychology. 2005; 24(6):811.

22. Vansteenkiste M, Sheldon KM. There's nothing more practical than a good theory: Integrating motivational interviewing and self-determination theory. British Journal of Clinical Psychology.2006; 45(1):63-82.

23. Deci EL, Ryan RM. The support of autonomy and the control of behavior. Journal of personality and social psychology.1987; 53(6):1024-37.

24. Flórez-Alarcón L. Investigación y diseminación de CEMA-PEMA-P: Un programa con enfoque motivacional breve para la prevención primaria del abuso de alcohol en estudiantes. In: Echeverría-Sanvicente y Carrascoza-Venegas C, editor. Diseminación de programas de prevención y tratamiento de conductas adictivas. México: UNAM. 2006.

25. Castellanos C. Efectos de la Entrevista Motivacional sobre la Motivación Autónoma en Jóvenes Consumidores de Alcohol. [Tesis de Maestria ]. Bogotá: Universidad Nacional de Colombia; 2009.

26. Sobell MB, Sobell LC.Guided self-change model of treatment for substance use disorders. Journal of Cognitive Psychotherapy.2005; 19(3):199-210.

27. Best JA, Thomson SJ, Santi SM, Smith EA, Brown KS. Preventing cigarette smoking among school children. Annual review of public health.1988; 9(1):61-201.

28. Becoña E. Bases Científicas de la Prevención de las Drogodependencias. Madrid: Delegación del Gobierno para el Plan Nacional sobre Drogas; 2002.

29. Ewing JA. Detecting alcoholism: the CAGE questionnaire. JAMA 1984; 252(14):905.

30. Oswald AL. L'utilité du questionnaire CAGE pour le dépistage de l'alcoolisme dans la santé publique [Tesis de Docteur en Médecine]: Université de Genève; 1999.

31. Flórez-Alarcón L, Vélez H, Jaimes M, Richart M. Diagnóstico sobre el consumo de alcohol y calidad de vida en estudiantes de secundaria del departamento de Casanare .Colombia y recomendaciones para la prevención selectiva e indicada del consumo excesivo. Boletín Electrónico de Salud Escolar, TIPICA. 2008; 4(1):1-35

32. Flórez-Alarcón L. Cuestionario de etapas integrado a un programa (CEMAPEMA) para la modificación del consumo abusivo de alcohol: Evaluación de etapas y de variables intermediarias. Acta Colombiana de Psicología.2003; 9:83-104.

33. Ryan RM, Connell JP. Perceived locus of causality and internalization: Examining reasons for acting in two domains. Journal of personality and social psychology.1989; 57(5):749-61

34. Miller WR, Yahne CE, Moyers TB, Martinez J, Pirritano M. A randomized trial of methods to help clinicians learn motivational interviewing. Journal of Consulting and Clinical Psychology. 2004; 72(6):1050-62.

35. Ryan RM, Plant RW, O'Malley S. Initial motivations for alcohol treatment: Relations with patient characteristics, treatment involvement, and dropout. Addictive behaviors.1995; 20(3):279-97. 